\title{
Changes in expression and production of heme oxygenase-1 in rats with acute liver injury induced by lipopolysaccharide
}

\author{
Juan Tang, Cheng-Min Li, Lian Li, Jie Wu and Gen-Lin Wang \\ College of Animal Science and Technology, Nanjing Agricultural University, Nanjing 210095, China
}

(Received February 20, 2016; Accepted May 13, 2016)

\begin{abstract}
To investigate the changes of heme oxygenase-1 (HO-1) expression and production in rats with acute liver injury induced by lipopolysaccharide (LPS), and explore the role of HO-1 in the pathogenesis of liver injury. Liver injury was assessed histologically and the serum level of alanine transaminase (ALT) and aspartate transaminase (AST) were examined. The activity of super oxide dismutase (SOD) and the content of malondialdehyde (MDA) and carbon monoxide (CO) in liver tissues were also examined at the same time. HO-1 mRNA expression was examined at different time points following LPS treatment and the expression of HO-1 protein was determined by immunohistochemical staining. Administration of LPS caused severe hepatic damage, characterized by significant elevation of serum ALT and AST levels and hepatic MDA content as well as a remarkable reduction of liver SOD activity at $24 \mathrm{hr}$ as compared with those in the control group. HO-1 activity was elevated significantly after modeling, showing a time-dependent manner from 6 to $24 \mathrm{hr}$, while expression of HO-1 protein was increased remarkably from 6 to $24 \mathrm{hr}$. Endogenous $\mathrm{CO}$ concentration in the liver of control rats remained very low but was elevated significantly after LPS treatment $(6,12,24 \mathrm{hr})$, which was in accordance with the changes of HO-1. HO-1 activity and protein are increased significantly in rats with acute liver injury induced by LPS, suggesting that HO-1 plays an important role in the pathogenesis of acute hepatic damage.
\end{abstract}

Key words: Lipopolysaccharide, Heme oxygenase-1, Acute injury, Liver, Rat

\section{INTRODUCTION}

Heme oxygenase-1 (HO-1), the rate-limiting enzyme in heme catabolism, has been shown to be induced during oxidative injury, and its induction acts as an important cellular defense mechanism against such injuries. It has been found that HO-1 serves as a protective gene by virtue of anti-inflammatory, anti-apoptotic and anti-oxidative actions and improves microcirculation in many cell types (Camara and Soares, 2005; Wagener et al., 2003; Otterbein et al., 2003). Amersi also reported that up-regulation of HO-1 protects genetically fat Zucker rat livers from ischemia/reperfusion injury (Amersi et al., 1999).

Oxidative stress, which refers to the in vivo oxidation and antioxidation imbalance, reflects an imbalance between the systemic manifestation of reactive oxygen species and a biological system's ability to readily detoxify the reactive intermediates or to repair the resulting damage (Hou et al., 2013; Sun et al., 2002). In tissues, the primary antioxidant enzymes responsible for protection from oxidative stress are superoxide dismutase
(SOD), malondialdehyde (MDA), and glutathione peroxidase (GPx) (Ceballos-Picot et al., 1992; Yasui and Baba, 2006). Apoptosis has been identified as a mechanism of hepatic injury, and caspase- 3 is probably the best understood of the mammalian caspases in terms of its specificity and roles in apoptosis. The activation of caspase-3 can be used as an improved index of apoptosis (Khan and Brown, 2002; Kam and Ferch, 2000).

Lipopolysaccharide (LPS) is an active component of gram-negative bacteria cell wall that causes activation of an oxidative stress response. Previous studies also have shown that HO-1 expression in the ovary has been shown to be essential for a proper oocyte ovulation, fertilization, and corpora lutea (Zenclussen et al., 2014). In addition, liver receptor homologue 1 has a multifunctional steroidogenic factor in ovarian physiology (Liu et al., 2003). However, the role of HO-1 in LPS-stimulated hepatocyte oxidative stress has rare reported. In this study, we performed a preliminary assessment on the adverse effects of acute LPS exposure on hepatic functions and concomitant elevations in HO-1 expression in liver tissues.

Correspondence: Gen-Lin Wang (E-mail: glwang@njau.edu.cn) 


\section{MATERIALS AND METHODS}

\section{Animals}

Adult male Sprague-Dawley rats (10-11 wk-of-age, 220$300 \mathrm{~g}$ ) were purchased from the Nanjing Qinglongshan Experimental Animal Factory (Nanjing, China) for use in this study. The rats were reared in separate cages under environmental conditions including free access to diet, 12-12 hr light-dark cycles, and a room temperature ranging from 23 to $25^{\circ} \mathrm{C}$. All experiment procedures were carried out in accordance with the National Institutes of Health Guidelines for Animal Care and approved by the local Ethics Committee.

\section{Animal treatment and tissue preparation}

Sixty-four Sprague-Dawley male rats were divided into five groups and injected intraperitoneally with LPS ( 0 or $10 \mathrm{mg} \cdot \mathrm{kg}^{-1}$ body weight, ip, $\mathrm{n}=8 /$ dosage; lipopolysaccharide from Escherichia coli 0111:B4 was purchased from Sigma Co., New York City, NY, USA) for $6,12,24$ or $36 \mathrm{hr}$ to establish the models of acute liver injury in male rats. LPS was prepared to $2.4 \mathrm{mg}$ / $\mathrm{mL}$ in normal saline solution; control rats were injected intraperitoneally with the same volume of saline. At $6,12,24$ or $36 \mathrm{hr}$ after the treatment with LPS, the rats were anesthetized with pentobarbital sodium ( $4 \mathrm{mg} / \mathrm{kg}$ body weight, iv) and then euthanized by cervical vertebra dislocation. After cervical vertebra dislocation, blood samples were collected from the jugular vein and placed at $37^{\circ} \mathrm{C}$ for $1 \mathrm{hr}$ before being centrifuged. The livers were dissected out immediately and washed with normal saline, dried on a filter paper. Then the livers were prepared immediately for further examinations. One piece of liver were fixed in 4\% paraformaldehyde, and embedded in paraffin for morphometric analysis.

Paraffin-embedded sections (5 $\mu \mathrm{m}$ thick) of the liver were transferred onto slides. Sections were stained with hematoxylin and eosin (HE) or immunohistochemistry. The homogenate of liver was centrifuged at 3,500 rpm for $10 \mathrm{~min}$, the supernatant was collected and the activities of caspase-3, super oxide dismutase (SOD) and malondialdehyde (MDA), carbon monoxide (CO) content were analyzed. All operations were done at $4{ }^{\circ} \mathrm{C}$.

\section{Immunohistochemistry and histology evaluation}

For immunohistochemical analysis, HO-1 expression was analyzed on formalin-fixed liver tissue. Paraffin-embedded sections ( $5 \mu \mathrm{m}$ thick) of the livers were transferred onto 3-aminopropyl-triethoxysilane (APES)-coated slides. Sections were deparaffinised with xylene and dehydrated through a graded ethanol series, rinsed in phosphate-buffered saline (PBS) and then incubated in $3 \% \mathrm{H}_{2} \mathrm{O}_{2}$ in PBS for 10 min to quench endogenous peroxidase. The tissue sections were quenched in normal goat serum for $30 \mathrm{~min}$ and incubated overnight at $4{ }^{\circ} \mathrm{C}$ with the primary antibody HO-1 (diluted 1:100) (Beyotime Biotechnology, China; diluted 1:100). Immunoreactivity was detected using biotinylated goat anti-rabbit IgG secondary antibody followed by avidin-biotinylated horseradish peroxidase complex visualized with diaminobenzidine tetrahydrochloride according to the manufacturer's instructions. Negative controls were obtained by omitting the primary antibodies. Sections were counterstained with hematoxylin and the stained section were observed and photographed using NIS-Elements BR $2.30(40 \times)$. For histological evaluation, the liver tissue sections were stained with HE. And then these sections were investigated under light microscopy.

\section{Biochemical indicators of liver function}

Blood samples were centrifuged at 3,500 rpm for $15 \mathrm{~min}$. Serum was collected and used to determine biochemical parameters: alanine aminotransferase (ALT) and aspartate aminotransferase (AST). The results were expressed as $\mathrm{U} / \mathrm{L}$.

\section{Superoxide dismutase activity}

The SOD activity was analyzed using commercial kits (NJBI, Nanjing, China) according to the method described by the manufacturer's instructions. The resulting absorbance of each sample was measured at $550 \mathrm{~nm}$ in a Nanodrop 8000 spectrophotometer (Thermo Fisher Scientific, Wilmington, DE, USA) and total SOD activity expressed as $\mathrm{U} / \mathrm{mg}$ protein.

\section{Malondialdehyde content}

Malondialdehyde (MDA) content in each homogenate was measured using the thiobarbituric acid (TBA) method at an absorbance of $532 \mathrm{~nm}$ and the results were expressed as nmole MDA/mg protein.

\section{CO content}

After centrifugation of the crude homogenates of liver tissue, endogenous $\mathrm{CO}$ content in the homogenates was determined using a Carbon Monoxide assay kit (NJBI, Nanjing, China) according to manufacturer instructions. The results were expressed as $\mu$ mole $\mathrm{CO} / \mathrm{g}$ tissue.

\section{Caspase-3 enzymatic activity}

Caspase-3 enzymatic activity was determined using a Caspase Apoptosis Assay Kit (Geno Technology Inc., St. Louis, MO, USA). 
HO-1 plays a protective role in rat liver

\section{Real time-PCR (RT-PCR)}

The level of HO-1 mRNA in the liver was evaluated by qualitative real-time PCR, which was performed using an ABI Prism Sequence Detection System (Applied Biosystems, New York City, NY, USA). Total RNA was extracted from approximately $100 \mathrm{mg}$ of frozen liver tissues using TRIzol reagent (Invitrogen, Carlsbad, CA, USA) and treated with DNase I (RNasefree) (TaKaRa, Dalian, China) to remove genomic DNA. The concentration and purity of the isolated total RNA was determined spectrophotometrically at 260 and $280 \mathrm{~nm}$ with a Nanodrop ${ }^{\circledR} 8000$ (Thermo Fisher Scientific). Total RNA from each sample was converted into cDNA with an Omniscript ${ }^{\circledR}$ Reverse Transcription kit (Takara) with Oligo-dT primers (Takara) and used for RT-PCR. SYBR Green (Applied Biosystems) real-time PCR Kit was used to measure mRNA expression of genes (HO-1 and caspase-3) expressed relative to the quantity of the $\beta$-actin endogenous control. Rat-specific primers were designed from published GenBank sequences and were synthesized by Sangon (Table 1). The fold differences in mRNA expression of samples were relative to the internal control sample, which was included in all runs.

\section{HO-1 quantitation by ELISA}

Tissue HO-1 protein levels were determined using a commercially available ELISA (enzyme linked immunosorbent assay) specific for the mice cytokine, purchased from R\&D Systems (Shanghai, China); this kit shows sensitivity of $0.78 \mathrm{ng} / \mathrm{mL}$ and ranges up to $25 \mathrm{ng} / \mathrm{mL}$.

\section{Statistical analysis}

All of the data are presented as the mean \pm standard deviation $(\mathrm{SD})$ and were analyzed by one-way analysis of variance (ANOVA) followed by Dunnett's multiple comparison test. The statistical analyses were performed with GraphPad Prismsoftware (GraphPad Software, San Diego, CA, USA). Differences were considered to be statistically significant when $\mathrm{P}<0.05$.

\section{RESULTS}

\section{Morphology of LPS exposed liver}

The liver histopathological changes are shown in Fig. 1. The control group did not show any morphological changes in the tissue (Fig. 1A). By contrast, the liver sections of LPS-treated rats indicated that LPS induced liver damage. These damages were apoptosis of some hepatocyte, focal necrosis, mononuclear cell infiltration, fatty degeneration and swelling of hepatocytes in liver tissue of treatment groups (Figs. 1B, C, D and E). Compared to the control group, after $6 \mathrm{hr}$ of LPS exposure, the rats showed apoptosis of some hepatocytes (Fig. 1B). After $12 \mathrm{hr}$ of LPS exposure, mononuclear cell infiltration, fatty degeneration and swelling of hepatocytes appeared (Fig. 1C). Liver injury peaked after $24 \mathrm{hr}$ of LPS exposure, characterized by greater levels of focal necrosis and fatty degeneration, and balloon degeneration of cells was also apparent in liver cells (Fig. 1D). However, only mild injury was present in the rat with little evidence of mononuclear cell infiltration and swelling of hepatocytes after $36 \mathrm{hr}$ of LPS-treatment (Fig. 1E).

\section{Assessment of liver function}

Hepatotoxicity was monitored by quantitative analysis of the serum ALT and AST activities, which were used as the biochemical markers of liver damages. It was observed that there were serious effects on activity of ALT and AST enzymes, indicating the hepatotoxicity induced by LPS (Fig. 2). Serum ALT level in the LPS exposure group was higher than in the control group and showed significant differences at 6,12 and 36-hr LPS treatment groups compared with the control group $(\mathrm{P}<$ 0.05 ), and this elevation persisted until $24 \mathrm{hr}$ after LPS treatment, which showed significant differences when compared with the control group ( $\mathrm{P}<0.01)$ (Fig. 2A). The level of AST in the LPS group started to increase at $6 \mathrm{hr}$ after LPS-treatment, but showed no significant differences when compared with the control group ( $\mathrm{P}>0$. 05). But, in the 12, 24 and 36-hr LPS exposure groups,

Table 1. Primer sequences for RT-PCR.

\begin{tabular}{lccl}
\hline Gene symbol & Accession No. & Product size (bp) & \multicolumn{1}{c}{ Primer sequence (5' to 3') } \\
\hline HO-1 & NM_012580.2 & 182 & F: AGGTGCACATCCGTGCAGAG \\
Caspase-3 & & $\begin{array}{l}\text { R: CTTCCAGGGCCGTATAGATATGGTA } \\
\text { F:AAGCCGAAACTCTTCATC }\end{array}$ \\
$\beta$-actin & NM_012922 & & $\begin{array}{l}\text { R:TGAGCATTGACACAATACAC } \\
\text { NM_031144 }\end{array}$ \\
& & 176 & F: CGTTGACATCCGTAAAGACC \\
\hline
\end{tabular}



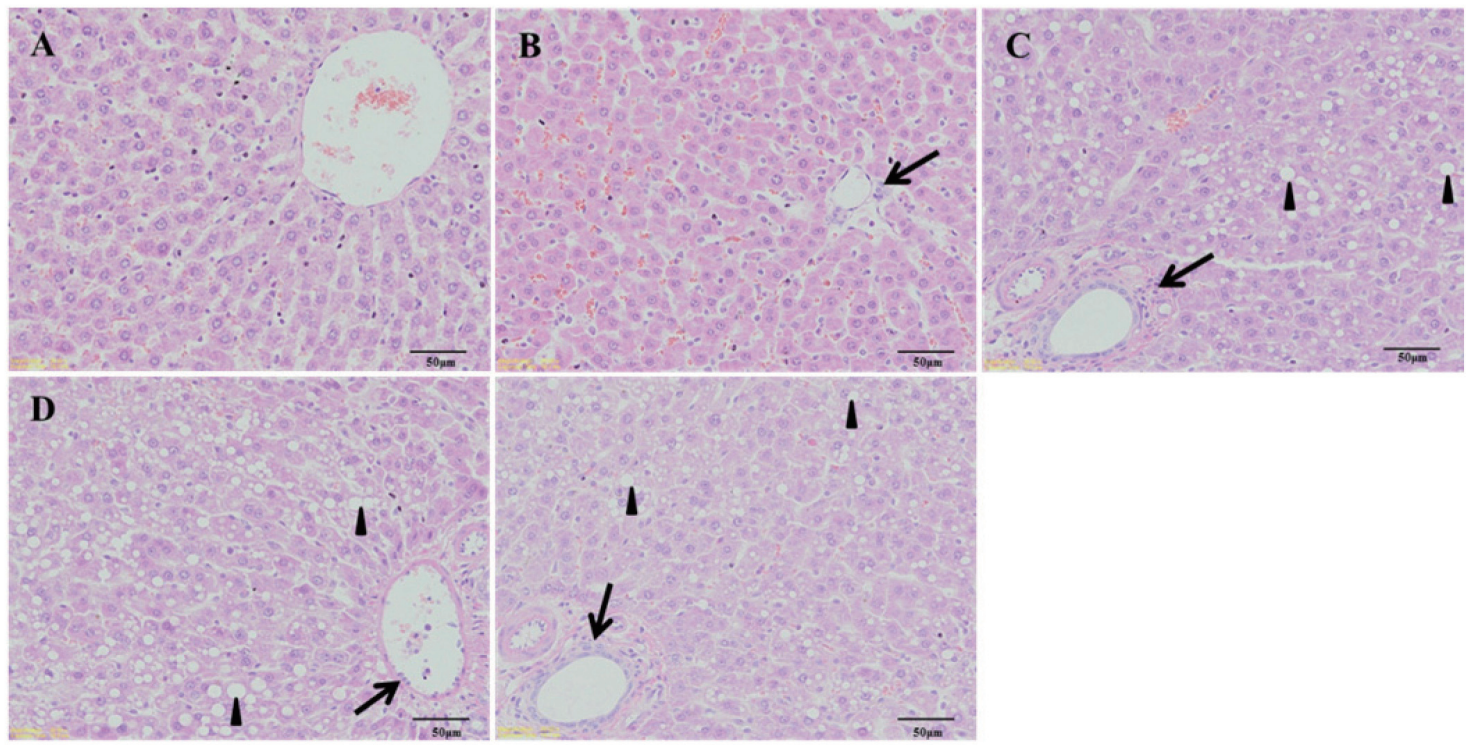

Fig. 1. Histological evaluation of the rat liver. Livers were harvested at 6,12,24 and $36 \mathrm{hr}$ after the LPS treatment and the sections were stained using H\&E. Representative sections from each treatment group are shown; $200 \times$ magnification). (A) No signs of liver damage were observed in the liver of controls. (B) Liver sections from 6-hr LPS treatment rats exhibited mild hepatocyte degeneration, apoptosis or necrosis. (C) Liver sections from 12-hr LPS treatment rats exhibited mild peri-portal expansion and fatty degeneration changes accompanied by a small amount of inflammatory cell infiltration and lipid droplets. (D) Liver sections from 24-hr LPS treatment rats exhibited severe damage to hepatocytes, which was demonstrated by the presence of greater levels of structure disorder, and there was also fatty degeneration and monocyte infiltration. (E) Liver sections from 36-hr LPS treatment rats showed that only mild injury was present in the rats with little evidence of mononuclear cell infiltration and swelling of hepatocytes. Arrowhead shows fatty degeneration. Arrow shows inflammatory cell infiltration.

AST levels were significantly higher when compared with the control group $(\mathrm{P}<0.05)$ (Fig. 2B). The control group showed no alteration after administration of the vehicle.

\section{MDA, CO content and Caspase-3, SOD activity in the liver tissue supernatant}

The MDA and CO content in the LPS group started to substantially increase at $12 \mathrm{hr}$ after LPS exposure, and this elevation persisted until $24 \mathrm{hr}$ after LPS treatment, which showed significant differences when compared with the control group $(\mathrm{P}<0.01)$. Meanwhile, at $36 \mathrm{hr}$ after LPS exposure, the MDA and CO content was also significantly increased when compared with the control group $(\mathrm{P}<0.01)$ (Figs. 3A and $\mathrm{B})$.

Caspase-3 activity was assessed after LPS exposure (Fig. 3C), and after 24 and $36 \mathrm{hr}$ of LPS exposure, we detected a significant increase in caspase- 3 activity compared with the control group $(\mathrm{P}<0.05)$. Caspase-3 activity also increased at the 6 and 12-hr LPS exposure time points, but it was not significant when compared with the control group $(\mathrm{P}>0.05)$.

In contrast, the hepatic SOD activity decreased significantly at $12 \mathrm{hr}$ after LPS exposure and bottomed out at
$24 \mathrm{hr}$ after LPS treatment. The 36-hr LPS exposure group also showed a significant decrease when compared with the control group $(\mathrm{P}<0.05)$ (Fig. 3D).

These effects in the LPS-administered group were induced by LPS itself because the control group showed no alteration after administration of the vehicle.

\section{HO-1 expression in the liver}

Hepatic HO-1 mRNA expression was significantly increased after LPS exposure for 12, 24 and $36 \mathrm{hr}$ compared to the control group and peaked at $24 \mathrm{hr}$ (Fig. 4A). At the same time, liver HO-1 protein levels in the LPS exposure group were also significantly increased compared with the control group after LPS exposure for 12, 24 and $36 \mathrm{hr}$ and peaked at $24 \mathrm{hr}$ (Fig. 4C). Moreover, localization of HO-1 protein expression was determined in liver tissues by immunohistochemical staining. HO-1 protein expression in hepatocytes was higher in the 24-hr LPS exposure group than in the other LPS treatment groups, which was shown as brown cytoplasmic staining around the central veins where the liver injury was more serious (Fig. 5). No difference was observed in the control group after treatment with the vehicle. 
HO-1 plays a protective role in rat liver
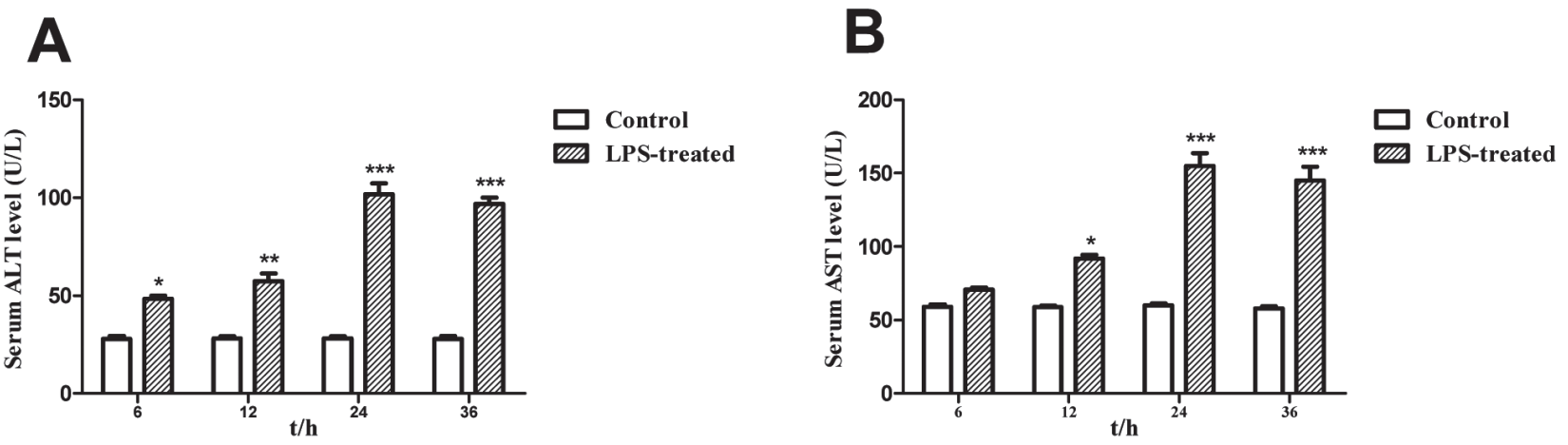

Fig. 2. Effect of different treatments on serum ALT and AST levels in the liver of rats. Animals were treated as described in materials and methods. (A) The level of ALT in the serum; (B) The level of AST in the serum; Data shown are mean \pm S.D. of eight animals in each group. Compared to control; *p $<0.05$ and $* * p<0.01$.
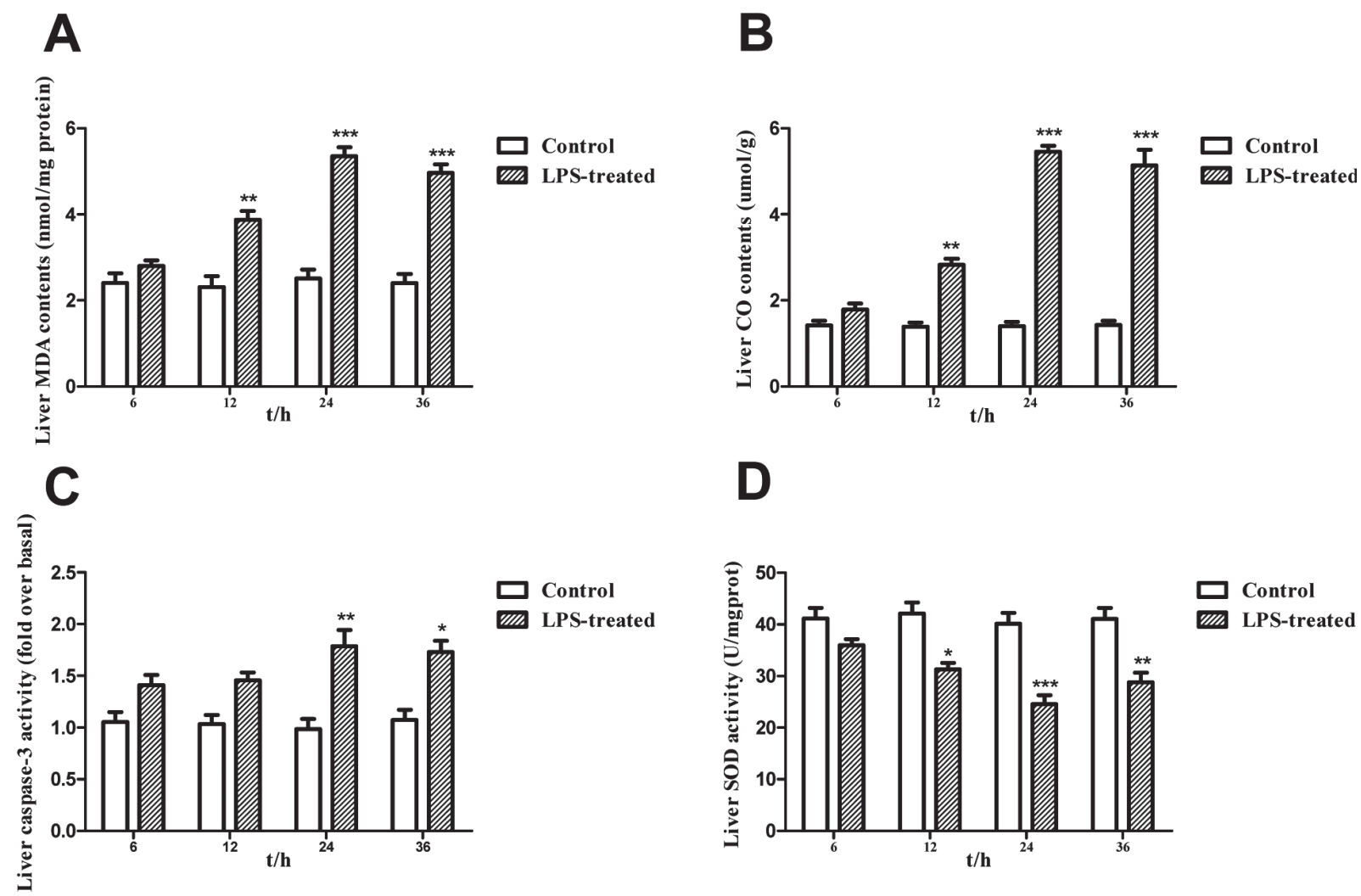

Fig. 3. Effect of different treatments on MDA and CO content, relative caspase-3 and SOD activities levels of rat liver. (A) The MDA content in the liver. (B) The CO content in liver; (C) Relative caspase-3 activity in liver; (D) The SOD activity in the liver. Data shown are mean \pm S.D. of eight animals in each group. Compared to control; * $p<0.05$ and $* * p<0.01$.

\section{Caspase-3 expression in liver}

The control group showed no change after administration of the vehicle. Compared with the control group, caspase-3 mRNA levels significantly increased after LPS exposure for 6, 12, 24 and $36 \mathrm{hr}$, but no significant differences were observed in caspase-3 mRNA levels after $6 \mathrm{hr}$ of LPS exposure $(\mathrm{P}>0.05)$. However, caspase-3 mRNA levels were significantly increased 
J. Tang et al.
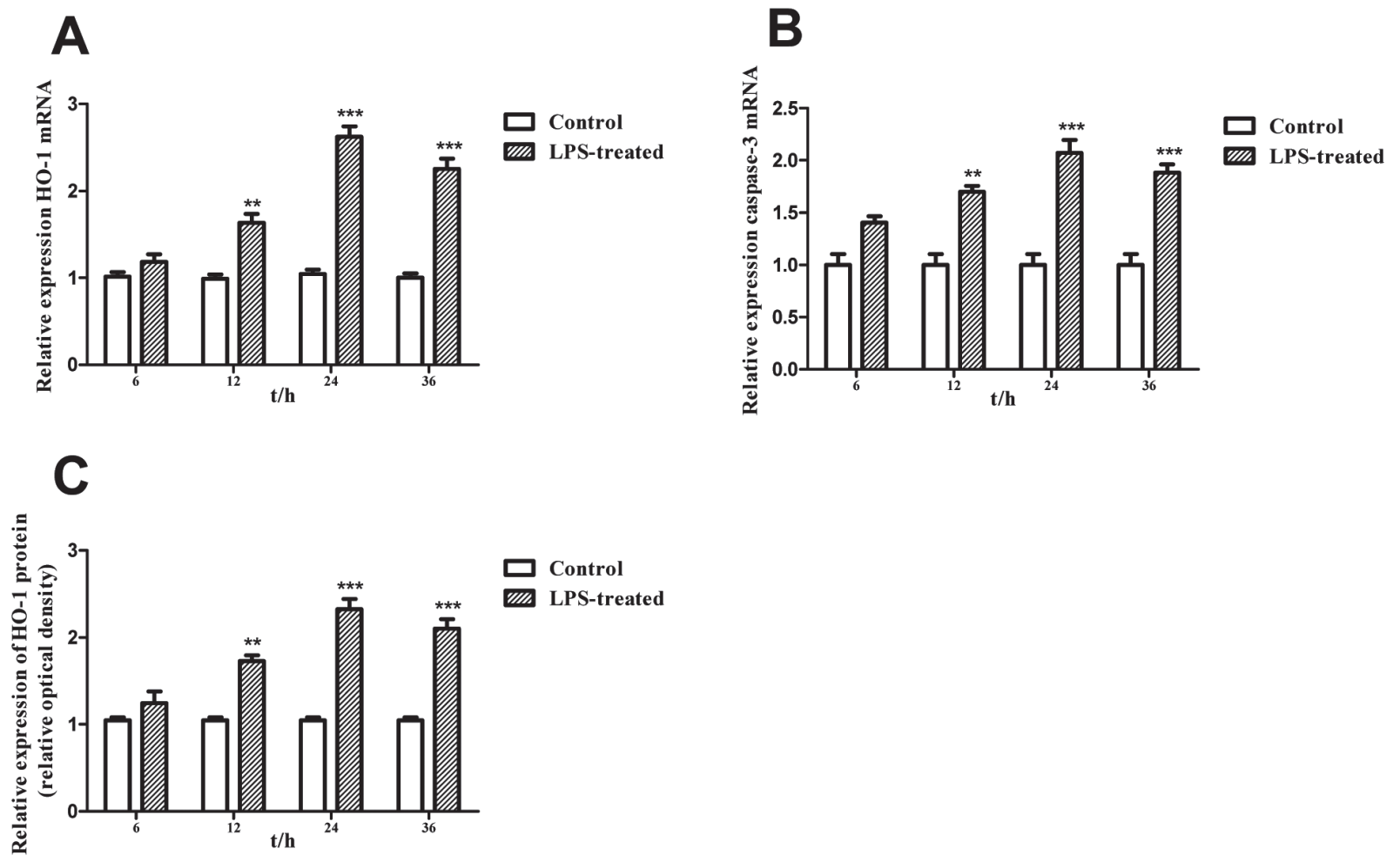

Fig. 4. Real-time RT-PCR analyses of HO-1, Caspase-3 and relative HO-1 protein of liver. (A) HO-1 mRNA (B) Caspase-3 mRNA. (C) relative HO-1 protein. Data shown are mean \pm S.D. of eight animals in each group. Compared to control; $* \mathrm{p}<0.05$ and $* * \mathrm{p}<0.01$.

after LPS exposure for 12, 24 and $36 \mathrm{hr}$ and peaked at $24 \mathrm{hr}$ compared with the control group $(\mathrm{P}<0.01)$ (Fig. 4B).

\section{DISCUSSION}

HO-1 is expressed ubiquitously and highly induced by numerous stress stimuli (Gozzelino et al., 2010), and plays protective roles by the regulation of antioxidant, anti-inflammatory, and anti-apoptotic activities (Li et al., 2015). CO and biliverdin, both generated via the catabolism of heme by the isoform HO-1, are potent immunosuppressors (Gozzelino et al., 2010). Meanwhile, recent evidence suggests that $\mathrm{CO}$ as a gaseous by-product of heme metabolism has long been thought to participate in many biological events and plays a pivotal role in mediating cytoprotection against oxidant-induced injury (Liu et al., 2010; Moore et al., 2005). LPS, a major component of gram-negative cell walls, is a potent immune-stimulator (Li et al., 2015). Lekić et al. (2011) analyzed oxidant/ antioxidant and apoptotic gene expressions in rats treated with D-galactosamine-lipopolysaccharide. Some studies also have clarified that LPS has effects on the reproductive functions of animals (Brecchia et al., 2014; Shimizu et al., 2012) and severe ovarian hyperstimulation syndrome was due to liver dysfunction (Balasch et al., 1990). However, the precise role of HO-1 in the protection of the liver subjected to LPS exposure and the underlying mechanisms still remains unclear.

The present study was undertaken to define the adverse effects of acute LPS exposure on hepatic functions and determine the association between stress-induced liver injury and concomitant elevations in HO-1 expression in liver tissues. We examined the effects of LPS on the liver tissue toxicity and induction of the oxidative stress in the rat. We found that LPS led to mononuclear cell infiltration and fatty degeneration in the liver. AST catalyzes the reversible transfer of an amino group between aspartate and glutamate, and as such, is an important enzyme in the amino acid metabolism (Hou et al., 2013). AST and ALT are commonly measured clinically as markers of liver health, and thus the enhanced AST and ALT activities in the serum after $6,12,24$ and $36 \mathrm{hr}$ of LPS treatment indicated that LPS could lead to the organ pathology changes 

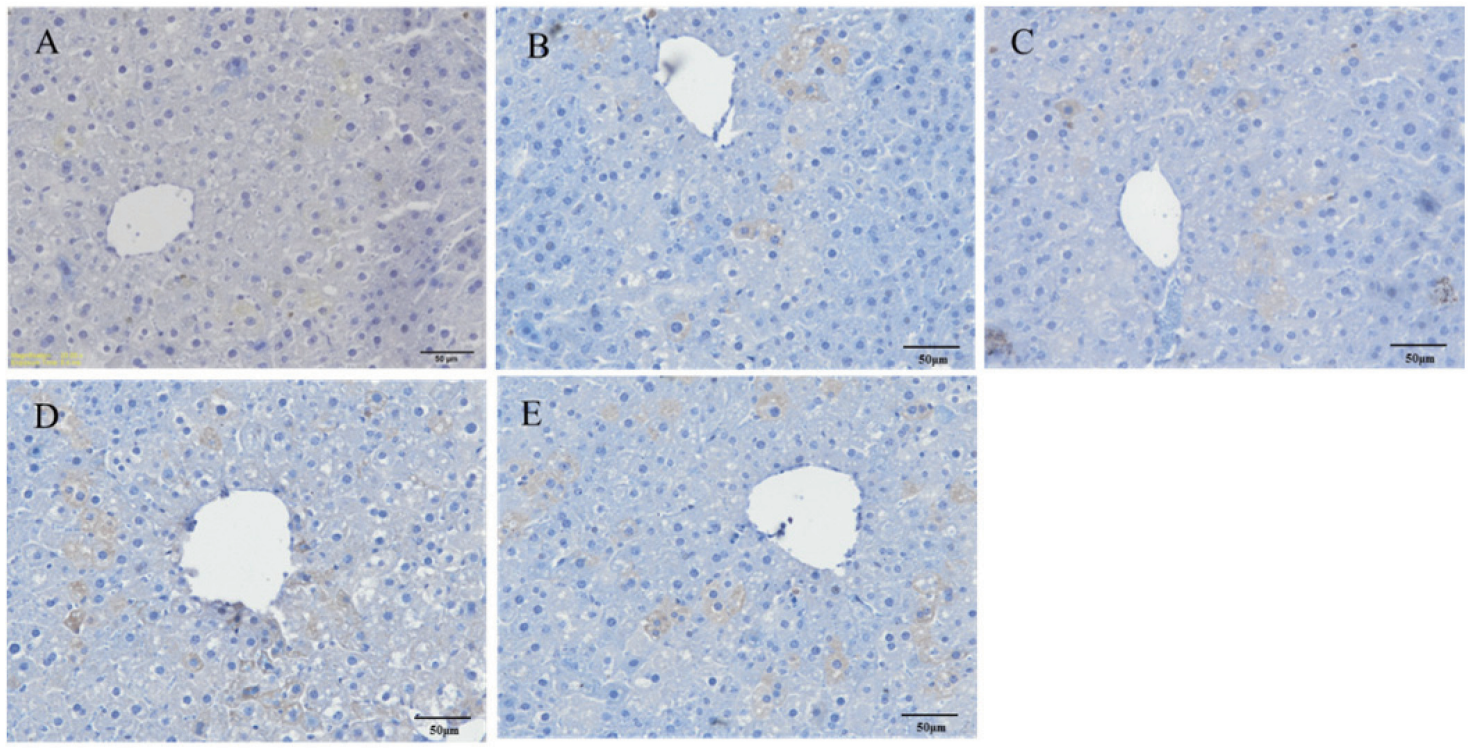

Fig. 5. Localization of HO-1 protein expression in liver tissues, shown as brown cytoplasmic staining, mainly around the central veins where the liver injury was more serious. (A) control group; (B) LPS-treated for $6 \mathrm{hr}$; (C) LPS-treated for $12 \mathrm{hr}$; (D) LPS-treated for $24 \mathrm{hr}$; (D) LPS-treated for $36 \mathrm{hr}$.

and liver damage, while the histopathological changes in the liver certified this deduction.

HO-1 is a stress-related, cytoprotective molecule that displays anti-apoptotic activity in various cells (Kirkby and Adin, 2006; Kiemer et al., 2003; Seo et al., 2014). $\mathrm{CO}$ as a gaseous by-product of heme metabolism has long been thought to participate in many biological events and plays a pivotal role in mediating cytoprotection against oxidant-induced injury (Amersi et al., 2002; Moore et al., 2005; Liu et al., 2010). Moreover, recent evidence suggests that the anti-apoptotic effect of $\mathrm{HO}-1$ has been attributed to its product $\mathrm{CO}$ in many types of cells (Al-Owais et al., 2012; Wang et al., 2011). Physiological concentration of $\mathrm{CO}$ is mainly derived from intrinsic heme degradation by HO-1 under stress conditions (Ryter et al., 2006). Furthermore, some studies indicated that free iron released by HO-1 caused mitochondrial dysfunction in pathological situations accompanied by endotoxic shock (Duvigneau et al., 2008). In the present study, the experiment showed that $\mathrm{CO}$ content was increased in livers of rats subjected to acute, transient LPS exposure, and hence it can be postulated that the protective effects of HO-1 may be mainly dependent on endogenous $\mathrm{CO}$, which deserves more studies in the future.

Caspases are crucial mediators of programmed cell death (apoptosis) with caspase-3 playing a central role in different apoptotic pathways. Moreover, the expression of caspase-3 is consistent with the change of hepatocyte apoptosis (Gao et al., 1998). Our results showed that caspase-3 in the LPS group was increased after LPS-treatment, which indicates that the LPS could lead to hepatocyte apoptosis.

MDA activity is an indicator of oxidative stress in both serum and liver. Previous work showed that LPS treatment resulted in the increased level of MDA in rat liver (Tang et al., 2015). Our in vivo result showed an increase pattern with significantly elaborated MDA level in the liver, which indicates that the LPS could lead to worse damage. SOD activity is one of the main parameters of antioxidative stress in the organism. LPS treatment decreases antioxidant enzyme activity in rat ovary (Li et al., 2015). Similarly, our results showed that SOD activities in the liver of the LPS-treated rats became reduced compared to the control group. The possible interpretation for this observation is that the metabolism of LPS mainly occurs in the liver, and these three anti-oxidative enzymes cooperate as follows: SOD catalyzes the dismutation of the superoxide radical $\left(\mathrm{O}^{2-}\right)$ to oxygen peroxide $\left(\mathrm{H}_{2} \mathrm{O}_{2}\right)$, protecting the organs from the $\mathrm{O}^{2-}$ damage. GPx catalyzes the reduction of $\mathrm{H}_{2} \mathrm{O}_{2}$ in water (Hou et al., 2013).

Taken together, the in vivo data presented here in rat model demonstrate that LPS could result in liver damage, elevated ASL and ALT activities in the serum, and increased MDA level in the liver tissues, indicative of 
oxidative stress. In addition, the results indicate that the over-expression of HO-1 possibly plays a protective role in the liver of LPS-treated rats, although the mechanistic basis of the hepatoprotective role of HO-1 has not yet been fully elucidated. Clearly, further investigations are required to clarify the possible protection mechanisms of HO-1 in LPS stressed liver.

\section{ACKNOWLEDGMENTS}

This work was supported by the National Supporting Projects for Science and Technology Sustentation Project of China (2012BAD12B10) and the Natural Science Foundation of China No.31372290.

Conflict of interest---- The authors declare that there is no conflict of interest.

\section{REFERENCES}

Al-Owais, M.M., Scragg, J.L., Dallas, M.L., Boycott, H.E., Warburton, P., Chakrabarty, A., Boyle, J.P. and Peers, C. (2012): Carbon monoxide mediates the anti-apoptotic effects of heme oxygenase-1 in medulloblastoma DAOY cells via $\mathrm{K}^{+}$channel inhibition. J. Biol. Chem., 287, 24754-24764.

Amersi, F., Buelow, R., Kato, H., Ke, B., Coito, A.J., Shen, X.D., Zhao, D., Zaky, J., Melinek, J., Lassman, C.R., Kolls, J.K., Alam, J., Ritter, T., Volk, H.D., Farmer, D.G., Ghobrial, R.M., Busuttil, R.W. and Kupiec-Weglinski, J.W. (1999): Upregulation of heme oxygenase-1 protects genetically fat Zucker rat livers from ischemia/reperfusion injury. J. Clin. Invest., 104, 16311639.

Amersi, F., Shen, X.D., Anselmo, D., Melinek, J., Iyer, S., Southard, D.J., Katori, M., Volk, H.D., Busuttil, R.W., Buelow, R. and Kupiec-Weglinski, J.W. (2002): Ex vivo exposure to carbon monoxide prevents hepatic ischemia/reperfusion injury through p38 MAP kinase pathway. Hepatology, 35, 815-823.

Balasch, J., Carmona, F., Llach, J., Arroyo, V., Jové, I. and Vanrell, J.A. (1990): Acute prerenal failure and liver dysfunction in a patient with severe ovarian hyperstimulation syndrome. Hum. Reprod., 5, 348-351.

Brecchia, G., Menchetti, L., Cardinali, R., Castellini, C., Polisca, A., Zerani, M., Maranesi, M. and Boiti, C. (2014): Effects of a bacterial lipopolysaccharide on the reproductive functions of rabbit does. Anim. Reprod. Sci., 147, 128-134.

Camara, N.O. and Soares, M.P. (2005): Heme oxygenase-1 (HO-1), a protective gene that prevents chronic graft dysfunction. Free Radic. Biol. Med., 38, 426-435.

Ceballos-Picot, I., Nicole, A., Clément, M., Bourre, J.-M. and Sinet, P.-M. (1992): Age-related changes in antioxidant enzymes and lipid peroxidation in brains of control and transgenic mice overexpressing copper-zinc superoxide dismutase. Mutation Research/DNAging, 275, 281-293.

Duvigneau, J.C., Piskernik, C., Haindl, S., Kloesch, B., Hartl, R.T., Hüttemann, M., Lee, I., Ebel, T., Moldzio, R., Gemeiner, M., Redl, H. and Kozlov, A.V. (2008): A novel endotoxin-induced pathway: upregulation of heme oxygenase 1 , accumulation of free iron, and free iron-mediated mitochondrial dysfunction.
Lab. Invest., 88, 70-77.

Gao, W., Bentley, R.C., Madden, J.F. and Clavien, P.A. (1998): Apoptosis of sinusoidal endothelial cells is a critical mechanism of preservation injury in rat liver transplantation. Hepatology, 27, 1652-1660.

Gozzelino, R., Jeney, V. and Soares, M.P. (2010): Mechanisms of cell protection by heme oxygenase-1. Annu. Rev. Pharmacol. Toxicol., 50, 323-354.

Hou, Y.-J., Zhao, Y.-Y., Xiong, B., Cui, X.-S., Kim, N.-H., Xu, Y.-X. and Sun, S.-C. (2013): Mycotoxin-containing diet causes oxidative stress in the mouse. PLoS One, 8, e60374.

Kam, P.C. and Ferch, N.I. (2000): Apoptosis: mechanisms and clinical implications. Anaesthesia, 55, 1081-1093.

Khan, V.R. and Brown, I.R. (2002): The effect of hyperthermia on the induction of cell death in brain, testis, and thymus of the adult and developing rat. Cell Stress Chaperones, 7, 73-90.

Kiemer, A.K., Gerwig, T., Gerbes, A.L., Meißner, H., Bilzer, M. and Vollmar, A.M. (2003): Kupffer-cell specific induction of heme oxygenase 1 (hsp32) by the atrial natriuretic peptide - role of cGMP. J. Hepatol., 38, 490-498.

Kirkby, K.A. and Adin, C.A. (2006): Products of heme oxygenase and their potential therapeutic applications. American Journal of Physiology-Renal Physiology, 290, F563-F571.

Lekić, N., Cerný, D., Hoř́nek, A., Provazník, Z., Martínek, J. and Farghali, H. (2011): Differential oxidative stress responses to D-galactosamine-lipopolysaccharide hepatotoxicity based on real time PCR analysis of selected oxidant/antioxidant and apoptotic gene expressions in rat. Physiol. Res., 60, 549-558.

Li, L., Tang, J., Sun, Y., Wu, J., Yu, P. and Wang, G. (2015): Upregulation of HO-1 Attenuates LPS-Stimulated Proinflammatory Responses Through Downregulation of p38 Signaling Pathways in Rat Ovary. Inflammation, 38, 1085-1092.

Liu, D.L., Liu, W.Z., Li, Q.L., Wang, H.M., Qian, D., Treuter, E. and Zhu, C. (2003): Expression and functional analysis of liver receptor homologue 1 as a potential steroidogenic factor in rat ovary. Biol. Reprod., 69, 508-517.

Liu, S.-H., Ma, K., Xu, X.-R. and Xu, B. (2010): A single dose of carbon monoxide intraperitoneal administration protects rat intestine from injury induced by lipopolysaccharide. Cell Stress Chaperones, 15, 717-727.

Moore, B.A., Overhaus, M., Whitcomb, J., Ifedigbo, E., Choi, A.M., Otterbein, L.E. and Bauer, A.J. (2005): Brief inhalation of lowdose carbon monoxide protects rodents and swine from postoperative ileus. Crit. Care Med., 33, 1317-1326.

Otterbein, L.E., Soares, M.P., Yamashita, K. and Bach, F.H. (2003): Heme oxygenase-1: unleashing the protective properties of heme. Trends Immunol., 24, 449-455.

Ryter, S.W., Alam, J. and Choi, A.M. (2006): Heme oxygenase-1/ carbon monoxide: from basic science to therapeutic applications. Physiol. Rev., 86, 583-650.

Seo, K., Yang, J.H., Kim, S.C., Ku, S.K., Ki, S.H. and Shin, S.M. (2014): The antioxidant effects of isorhamnetin contribute to inhibit COX-2 expression in response to inflammation: a potential role of HO-1. Inflammation, 37, 712-722.

Shimizu, T., Miyauchi, K., Shirasuna, K., Bollwein, H., Magata, F., Murayama, C. and Miyamoto, A. (2012): Effects of lipopolysaccharide (LPS) and peptidoglycan (PGN) on estradiol production in bovine granulosa cells from small and large follicles. Toxicology In Vitro, 26, 1134-1142.

Sun, Y., Zhang, J., Lu, L., Chen, S.S., Quinn, M.T. and Weber, K.T. (2002): Aldosterone-induced inflammation in the rat heart: role of oxidative stress. Am. J. Pathol., 161, 1773-1781. 
HO-1 plays a protective role in rat liver

Tang, J., Li, L., Li, C.M., Wu, J., Sun, Y. and Wang, G.L. (2015): Upregulation of HO-1 with Haemin Alleviates LPS-Stimulated Pro-inflammatory Responses Through Downregulation of p38 Signalling Pathways in Rat Liver. Scand. J. Immunol., 82, 443451.

Wagener, F.A., Volk, H.-D., Willis, D., Abraham, N.G., Soares, M.P., Adema, G.J. and Figdor, C.G. (2003): Different faces of the heme-heme oxygenase system in inflammation. Pharmacol. Rev., 55, 551-571.

Wang, X., Wang, Y., Lee, S.-J., Kim, H.P., Choi, A.M. and Ryter,
S.W. (2011): Carbon monoxide inhibits Fas activating antibodyinduced apoptosis in endothelial cells. Med. Gas Res., 1, 8.

Yasui, K. and Baba, A. (2006): Therapeutic potential of superoxide dismutase (SOD) for resolution of inflammation. Inflam.Res., 55, 359-363.

Zenclussen, M.L., Casalis, P.A., Jensen, F., Woidacki, K. and Zenclussen, A.C. (2014): Hormonal fluctuations during the estrous cycle modulate heme oxygenase- 1 expression in the uterus. Front. Endocrinol., 5, 32. 